\title{
Zur Theorie der Möbiusschen $\mu$-Funktion
}

von

W. Staś (Poznań)

1. G. H. Hardy und J. E. Littlewood haben gezeigt ([1], Seite 156-162) daß

$$
\sum_{k=1}^{\infty} \frac{(-x)^{k}}{k ! \zeta(2 k+1)}=O\left(x^{-1 / 4}\right)
$$

mit der Riemannschen Vermutung gleichwertig ist (vergl. [5], Seite 328). Das wesentlich interessante ist hier, daß die Bedingung (1.1) nur von den Werten explizite abhängt, die $\zeta(s)$ rechts von der Gerade $\sigma=1$ annimmt.

Schreiben wir (1.1) in der Gestalt

$$
\sum_{n=1}^{\infty} \frac{\mu(n)}{n} e^{-(\beta / n)^{2}}=O\left(\frac{1}{\sqrt{/}}\right),
$$

wo $\mu(n)$ die bekannte Möbiussche $\mu$-Funktion bezeichnet (Siehe [1], Seite 160).

Ich beabsichtige in dieser Publikation eine explizite, numerische, nntere Abschätzung von (1.2) mit Turánschen Methoden zu finden.

Es bezeichne

$$
M(x)=\sum_{n \leqslant x} \mu(n)
$$

Wir werden den folgenden Satz zeigen:

SATz. Wenn

$$
\int_{i}^{T}\left(\frac{M(x)}{x}\right)^{2} d x<\alpha \log T,
$$

dann gilt für

$$
T>\max \left(c_{0}, e^{\sqrt{\alpha}}\right)
$$


die folgende Abschätzung

$$
\max _{T e^{-\varphi(T)} \leqslant \beta \leqslant T}\left|\sum_{n=1}^{\infty} \frac{\mu(n)}{n} e^{-(\beta / n)^{2}}\right| \geqslant \frac{1}{\sqrt{T}} e^{-3 \frac{\log T^{\prime} \log \log \log \log T}{\log \log T}} ;
$$

$\varphi(T)=\log T \log \log \log T / \log \log T$ und $c_{0}$ bezeichnet eine explizite numerische Konstante.

Beim Beweis wird das folgende Lemma von P. Turán ausgenutzt ([6], Seite 56, Korollar I).

LEMMa. Wenn $z_{1}, z_{2}, \ldots, z_{M}$ von einander und von 0 verschiedene komplexe Zahlen bedeuten, für welche $\max \left|z_{j}\right|=1$, mit $\min \left|z_{\mu}-z_{p}\right| \geqslant \delta$, dann existiert bei beliebigem ganzen $m$ ein ${ }^{j}$ ganzes $\nu$ mit $m+{ }^{\mu \neq \nu} \leqslant \nu \leqslant m+M$ so, $d a \beta$

$$
\frac{\left|b_{1} z_{1}^{\nu}+\ldots+b_{M} z_{M}^{\nu}\right|}{\sum_{j=1}^{M}\left|b_{j}\right|\left|z_{j}\right|^{\nu}} \geqslant \frac{\delta^{M-1}}{M 2^{M}}
$$

Es wäre interessant die Voraussetzung (1.4) die eigentlich schärfer als die Riemannsche Vermutung ist, abzuschwächen. In diesem Fall mußte aber die Beweismethode etwas anders sein. Man mußte scheinbar den Turánschen Satz X anwenden ([6], Seite 52). Auf dieses Problem möchte ich noch zurückkommen.

2. Aus (1.4) folgt bekanntlich die Riemannsche Vermutung (Vergl. [3], Satz 477, Seite 154). Es sind also die folgenden Sätze anwendbar:

Für jedes $T \geqslant c_{1}$, gibt es ein solches $t_{T}, T<t_{T^{\prime}}<T+1$, daß auf $-1 \leqslant \sigma \leqslant 2$

$$
\frac{1}{\left|\zeta\left(\sigma+i t_{T}\right)\right|} \leqslant T^{c_{2}}
$$

gilt ([5], Theorem 14.16, Seite 303, [2]).

Bei festem $\eta$, auf $0<\eta<2$, für $t \geqslant 11$ gilt

([3], Seite 164).

$$
\frac{1}{\left|\zeta\left(\frac{1}{2}+\eta+i t\right)\right|} \leqslant t^{\frac{c_{3}}{\eta \log ^{\eta / 8} t}}
$$

Aus (1.4) folgt außerdem:

(2.3) Alle nichttrivialen Nullstellen von $\zeta(s)$ sind einfach

([5], Theorem 14.16, Seite 303).

$$
\text { Es gilt }
$$

$$
\frac{1}{\left|\zeta^{\prime}(\varrho)\right|} \leqslant \sqrt{\alpha}|\varrho|
$$

wo $\varrho$ die komplexen Nullstellen von $\zeta(s)$ bezeichnet ([5], [2]).
Wenn $\varrho_{1}, \varrho_{2}$ zwei verschiedene komplexe Nullstellen von $\zeta(s)$ bezeichnet, dann gilt

([5], Theorem 14.31, Seite 326, [2]).

$$
\left|\varrho_{1}-\varrho_{2}\right| \geqslant \frac{1}{15 \gamma^{/ \bar{\alpha}\left(\max _{i=1,2}\left|\varrho_{i}\right|\right)^{4}}}
$$

Bevor wir zum Beweis von (1.6) kommen, stellen wir einige Sätze über $\Gamma(s)$ und $\zeta(s)$ zusammen, welche im folgenden notwending sind.

Es gilt für die Eulersche $\Gamma$-Funktion

$$
|\Gamma(\sigma+i t)|=\frac{k \Gamma(1+\sigma)}{\sqrt{\sigma^{2}+t^{2}}} \sqrt{\frac{2 \pi t}{e^{\pi t}-e^{-\pi t}}}
$$

und $1 \leqslant k \leqslant 1 \overline{1+t^{2}}, 0<\sigma<1$ ([4], Seite 25$)$.

Bezeichnen wir mit $N(\tau)$ die Anzahl der Nullstellen von $\zeta(s)$ im $0<\sigma<1$, $0<t \leqslant \tau, \tau \geqslant 2$ dann gilt offenbar"

$$
N(\tau+1)-N(\tau)<c_{4} \log \tau
$$

Es gilt die folgende Funktionalgleichung der Riemannschen $\zeta$-Funktion:

([5], Seite 22).

(2.9) Die ersten komplexen Wurzeln von $\zeta(s)$ sind $\frac{1}{2} \pm i 14,13 \ldots$

([5], Seite 330).

3. Sei $T \geqslant 1000$. Wir führen folgende Bezeichnungen ein:

$$
\begin{aligned}
\varepsilon & =(\log \log T)^{-1}, \\
\omega & =2 \log T, \\
\omega_{0} & =2 \log T \log \log \log T(\log \log T)^{-1} .
\end{aligned}
$$

Für die ganze Zahl $k$ setzen wir voraus, daß sie die folgende Ungleichung erfüllt:

$$
(1-\varepsilon) \frac{\log T}{\log \log T} \leqslant k \leqslant \frac{\log T}{\log \log T} .
$$

Es bezeichne weiter

$$
l=\frac{\log T}{(\log \log T)^{4}} .
$$

4. Wenden wir auf (1.2) die bekannte Formel von Cahen-Mellin. Daraus ergibt sich unsere Ausgangsformel.

$$
\beta \sum_{n=1}^{\infty} \frac{\mu(n)}{n} e^{-(\beta / n)^{2}}=\frac{1}{2 \pi i} \int_{\left(\frac{1}{2}+\varepsilon\right)} \beta^{2 s} \frac{\Gamma\left(\frac{1}{2}-s\right)}{\zeta(2 s)} d s,
$$

$\beta>0, s=\sigma+i t([1]$, Seite 158). 
Setzen wir

$$
\beta=e^{\omega_{1} / 2}
$$

und integrieren (4.1) k-mal, von $\omega-\omega_{0}$ bis $\omega_{2}, \omega_{3}, \ldots, \omega_{k}, \omega$. Bezeichnen wir die Summe in (4.1) mit $H(\beta)$. Dann gilt nach der Integration:

$$
\begin{aligned}
I_{\omega} & =\int_{\omega-\omega_{0}}^{\omega} \int_{\omega-\omega_{0}}^{\omega_{k}} \ldots \int_{\omega-\omega_{0}}^{\omega_{3}} \int_{\omega-\omega_{0}}^{\omega_{2}}\left\{e^{\omega_{1} / 2} H\left(e^{\omega_{1} / 2}\right)\right\} d \omega_{1} \ldots d \omega_{k} \\
& =\frac{1}{2 \pi i} \int_{\left(\frac{1}{2}+\varepsilon\right)} \frac{e^{\omega s} \Gamma\left(\frac{1}{2}-s\right)}{s^{k} \zeta(2 s)} d s-\frac{1}{2 \pi i} \sum_{j=1}^{k} \frac{\omega_{0}^{k-j}}{(k-j) !} \int_{\left(\frac{1}{2}+\varepsilon\right)} \frac{e^{\left(\omega-\omega_{0}\right) s} \Gamma\left(\frac{1}{2}-s\right)}{s^{j} \zeta(2 s)} d s .
\end{aligned}
$$

Einerseits hat man einfach

$$
I_{\omega} \leqslant \frac{\omega_{0}^{k}}{k !} \max _{\omega-\omega_{0} \leqslant \omega_{1} \leqslant \omega}\left|e^{\omega_{1 / 2}} H\left(e^{\omega_{1} / 2}\right)\right|
$$

Durch geeignete Konturintegration und Grenzübergang bekommt man

(4.5) $\frac{1}{2 \pi i} \int_{\left(\frac{1}{2}+\varepsilon\right)} \frac{e^{\omega s} \Gamma\left(\frac{1}{2}-s\right)}{s^{k} \zeta(2 s)} d s=\frac{1}{2 \pi i} \int_{\left(\frac{1}{4}-\varepsilon\right)} \frac{e^{\omega s} \Gamma\left(\frac{1}{2}-s\right)}{s^{k} \zeta(2 s)} d s+2^{k-1} \sum_{\varrho}^{\top} \frac{e^{\omega \varrho / 2} \Gamma\left(\frac{1}{2}-\frac{1}{2} \varrho\right)}{\varrho^{k} \zeta^{\prime}(\varrho)}$.

Der Beweis von (4.5) folgt aus (2.2), (2.1), (2.3), (2.6) und (2.8). Aus (4.3) und (4.5) hat man nun

$$
\begin{aligned}
I_{\omega}=\frac{1}{2 \pi i} \int_{\left(\frac{1}{2}-\varepsilon\right)} \frac{e^{\omega s} \frac{\Gamma\left(\frac{1}{2}-s\right)}{s^{k} \zeta(2 s)} d s}{}+2^{k-1} \sum_{\varrho} \frac{e^{\omega e / 2} \Gamma\left(\frac{1}{2}-\frac{1}{2} \varrho\right)}{\varrho^{k} \zeta^{\prime}(\varrho)}- \\
-\frac{1}{2 \pi i} \sum_{j=1}^{k} \frac{\omega_{0}^{k-j}}{(k-j) !} \int_{\left(\frac{1}{2}+\varepsilon\right)} \frac{e^{\left(\omega-\omega_{0}\right) s} \Gamma\left(\frac{1}{2}-s\right)}{s^{j} \zeta(2 s)} d s .
\end{aligned}
$$

Die Summe in (4.6) teilen wir jetzt in zwei Summen

$$
\sum_{e}(\ldots)=\sum_{|\Im \rho|>l}(\ldots)+\sum_{\left|\Im_{0}\right| \leqslant l}(\ldots)
$$

wo $l$ mit (3.5) bestimmt ist.

Aus der Abschätzung der ersten Teilsumme, wegen (2.4), (2.6) und (2.7) ergibt sich

$$
\left|2^{k-1} \sum_{\left|\Omega_{e}\right|>l}(\ldots)\right| \leqslant c_{5} \sqrt{\alpha} \frac{2^{k} e^{\omega / 4}}{(k-1)([l]-1)^{k-1}}
$$

und wegen (3.2), (3.4) und (3.5) endlich

$$
\leqslant \sqrt{\alpha} e^{-3 \frac{\log T \log \log \log T}{\log \log T}}
$$

Wir werden jetzt die Integrale in (4.6) explizite abschätzen müssen. Der Integrationsweg des zweiten Integrals in (4.6) läßt sich bis zu der Gerade $\sigma=\frac{1}{4}+\varepsilon$ verschieben (Vergl. die Bemerkungen betreffs (4.5)). Man hat also

$$
\begin{aligned}
& \left|\int_{(1+r)} \frac{e^{\left(\omega-\omega_{0}\right) s} \Gamma\left(\frac{1}{2}-s\right)}{s^{j} \zeta(2 s)} d s\right| \\
& \leqslant e^{\left(\omega-\omega-\omega_{0}\right)\left(\frac{1}{4}+\varepsilon\right)} \int_{-\infty}^{+\infty} \frac{\left|\Gamma\left(\frac{1}{4}-\varepsilon-i t\right)\right|}{\left(t^{2}+\left(\frac{1}{4}+\varepsilon\right)^{2}\right)^{1 / 2}\left|\zeta\left(\frac{1}{2}+2 \varepsilon+2 i t\right)\right|} d t \\
& \leqslant 2 e^{\left(10-\omega_{0}\right)\left(\frac{1}{4}+s\right)}\left(\int_{0}^{11}+\int_{11}^{p(g)}+\int_{\varphi(c)}^{\infty}\right)
\end{aligned}
$$

$\varphi(\varepsilon) m u ß$ so gewählt sein, damit für $t \geqslant \varphi(\varepsilon)$, die Ungleichung

$$
\frac{c_{3}}{t^{2 e \log ^{2 \varepsilon / 3} t}} \leqslant e^{\frac{1}{4} \pi t}
$$

erfïlllt war. Es genügt ersichtlich

$$
\varphi(\varepsilon)=\left(\frac{c_{6}}{\varepsilon}\right)^{2}
$$

zu setzen; also wegen (3.1)

zu wählen.

$$
\varphi(\varepsilon)=\left(c_{6} \log \log T\right)^{2}
$$

Aus (2.2), (2.6) und (2.9) bekommt man einfach für $T>c_{10}$

$$
\int_{\varphi(e)}^{\infty}(\ldots) d t \leqslant c_{8}, \quad \int_{0}^{11}(\ldots) d t \leqslant c_{9}
$$

und endlich wegen (2.2), (2.6) und (4.9), für $T>c_{11}$

$$
\int_{11}^{\varphi(8)}(\ldots) d t \leqslant T^{\frac{(\log \log T)^{2}(\log \log \log T)^{2}}{\log T}} .
$$

Man hat daraus für $T>c_{12}$

$$
\left|\int_{\left(\frac{1}{+}+\varepsilon\right)} \frac{e^{\left(\omega-\omega_{0}\right) s} \Gamma\left(\frac{1}{2}-s\right)}{s^{j} \zeta(2 s)} d s\right| \leqslant e^{\left(\omega-\omega_{0}\right)(1+B)} T^{\frac{(\log \log T)^{2}(\log \log \log T)^{2}}{\log T}}
$$

und endlich

$$
\begin{aligned}
& \text { (4.11) }\left|\frac{1}{2 \pi i} \sum_{j=1}^{k} \frac{\omega_{0}^{k-j}}{(k-j) !} \int_{(1+\varepsilon)} \frac{e^{\left(\omega-\omega_{0}\right) 8} \Gamma\left(\frac{1}{2}-s\right)}{s^{j} \zeta(2 s)} d s\right| \\
& \leqslant c_{13} k\left(\frac{e \omega_{0}}{k}\right)^{k} e^{\left(\frac{1}{t}+\varepsilon\right)\left(\omega-\omega_{0}\right)} T^{\frac{(\log \log T)^{2}(\log \log \log T)^{8}}{\log T}} . \\
& \leqslant c_{14} \sqrt{T} e^{-\frac{1 \log T \log \log \log T}{\log \log T}} .
\end{aligned}
$$


Auf ähnlichem Weg folgt aus (2.2), (2.6) und (2.8) die Abschätzung des ersten Integrals in (4.6):

$$
\left|\frac{1}{2 \pi i} \int_{\left(\frac{1}{4}-\delta\right)} \frac{e^{\omega s} I^{\top}\left(\frac{1}{2}-s\right)}{s^{k} \zeta(2 s)} d s\right| \leqslant c_{15} e^{(1-\varepsilon) \omega} T^{\frac{\left(\log \log T^{T}\right)^{2}\left(\log \log \log T^{\prime}\right)^{2}}{\log T}}
$$

und endlich wegen (3.2)

$$
\leqslant c_{16} \cdot T e^{-\frac{\log T}{\log \log T}}
$$

Aus (4.4), (4.6), (4.8), (4.11) und (4.12) hat man nun

$$
\begin{aligned}
& \left|2^{k-1} \sum_{|\Im \varrho| \leqslant l} \frac{e^{\omega \varrho / 2} T\left(\frac{1}{2}-\frac{1}{2} \varrho\right)}{\varrho^{k} \zeta^{\prime}(\varrho)}\right| \leqslant \frac{\omega_{0}^{k}}{k !} \max \left|\epsilon^{\left(\omega_{1} / 2\right.} H\left(e^{\left(\omega_{1} / 2\right.}\right)\right|+ \\
& +\sqrt{\alpha} e^{\frac{3 \log T \log \log \log T}{\log \log T}}+c_{17} \sqrt{T} e^{-\frac{\log T}{\log \log T}}+c_{18} \sqrt{T} e^{-\frac{1 \log T \log \log \log T}{\log \log T}} .
\end{aligned}
$$

Bezeichnen wir mit $\varrho_{1}$ die erste komplexe Wurzel von $\zeta(s)$. Nach (2.9) hat man, mit $L$ die linke Seite von (4.13) bezeichnend:

$$
L=2^{k-1} \frac{e^{\omega / 4}}{\left|\frac{1}{2}+i 14,13 \ldots\right|}\left|\sum_{\mid\{\varrho \mid \leqslant l} \frac{\Gamma\left(\frac{1}{2}-\frac{1}{2} \varrho\right) e^{\frac{1}{2} \omega\left(\varrho-e_{1}\right)}}{\zeta^{\prime}(\varrho)}\left(\frac{\varrho_{1}}{\varrho}\right)^{k}\right|
$$

Sei $Z$ die Summe in (4.14). Wir schätzen $|Z|$ von unten mit Hilfe des Turánschen Lemmas (1.7) ab.

Wir setzen

$$
\begin{aligned}
& b_{\mu}=\frac{\Gamma\left(\frac{1}{2}-\frac{1}{2} \varrho_{\mu}\right) e^{\frac{1}{2} \omega\left(\varrho_{\mu}-\varrho_{1}\right)}}{\zeta^{\prime}\left(\varrho_{\mu}\right)} \\
& z_{\mu}=\frac{\varrho_{1}}{\varrho_{\mu}}
\end{aligned}
$$

Die Bedingung $\max \left|z_{\mu}\right|=1$, ist wegen (4.16) sicherlich erfüllt. Wir müssen jetzt den Wert des in Lemma (1.7) vorkommenden $\delta$ feststellen. Aus (2.5) und (2.9) hat man

$$
\left|z_{\mu}-z_{\nu}\right|=\frac{\left|\varrho_{1}\right|}{\left|\varrho_{\mu}\right|\left|\varrho_{\nu}\right|}\left|\varrho_{\nu}-\varrho_{\mu}\right| \geqslant \frac{\left|\frac{1}{2}+i 14,13 \ldots\right|}{\left|\frac{1}{2}+i \gamma_{\mu}\right|\left|\frac{1}{2}+i \gamma_{\nu}\right|} \cdot \frac{1}{15 \gamma^{/ \alpha}} \cdot \frac{1}{\max \left(\left|\varrho_{\mu}\right|,\left|\varrho_{\nu}\right|^{4}\right.} .
$$

Aus $|\mathfrak{S} \varrho| \leqslant l$ und $(3.5)$ hat man weiter für $T>\max \left(c_{20}, e^{l^{\alpha}}\right)$,

$$
\left|z_{\mu}-z_{\nu}\right| \geqslant \frac{\sqrt{\frac{1}{4}+14^{2}}}{15 \sqrt{\alpha}} \cdot \frac{1}{\left(\sqrt{\frac{1}{4}+l^{2}}\right)^{6}} \geqslant \frac{(\log \log T)^{23}}{\log ^{7} T} \stackrel{\text { Def }}{=} \delta .
$$

Aus Satz (1.7) folgt

$$
|Z| \geqslant \frac{\delta^{M-1}}{M 2^{M}} \sum_{j=1}^{M}\left|b_{j}\right|\left|z_{j}\right|^{k} \geqslant \frac{\delta^{M-1}}{M 2^{M}}\left|b_{1}\right|,
$$

wo $k$ die Bedingung

$$
m+1 \leqslant k \leqslant m+M
$$

erfïllt. Wir wählen $m$ in Ưbereinstimmung mit (3.4), also

$$
m=(1-\varepsilon) \frac{\log T}{\log \log T}-1 \text {. }
$$

Wegen (2.7) und (3.5) ist die Anzahl der Nullstellen ron $\zeta(s)$ im $0<\sigma<1,0 \leqslant t \leqslant l, I>c_{21}$,

Man kann also

$$
N(l)=\frac{\log T}{(\log \log T)^{3}} .
$$

$$
M=\frac{\log T}{(\log \log T)^{3}}
$$

setzen und damit ist die Bedingung für $k$ erfüllt.

Jetzt müßen wir das $\left|b_{1}\right|$ in (4.18) ausrechnen. Wegen $(2.3)$ und (2.6)

$\begin{aligned} & \text { hat man } \\ & (4.20)\end{aligned}\left|b_{1}\right|=\frac{\left|I^{\prime}\left(\frac{1}{2}-\frac{1}{2} \varrho_{1}\right)\right|}{\left|\zeta^{\prime}\left(\varrho_{1}\right)\right|}=\frac{\left|\Gamma\left(\frac{1}{4}-i 7,065 \ldots\right)\right|}{\left|\zeta^{\prime}\left(\varrho_{1}\right)\right|}=c_{22} \neq 0$.

Aus $(4.18),(4.17),(4.19)$ und (4.20) folgt einfach für $T>c_{23}$

$$
|z| \geqslant c_{22} \frac{\left\{\frac{(\log \log T)^{23}}{\log T}\right\}^{\frac{\log T}{(\log \log T)^{3}}-1}}{\frac{\log T}{(\log \log T)^{3}} 2^{\frac{\log T}{(\log \log T)^{2}}}} \geqslant e^{-4 \frac{\log T}{(\log \log T)^{2}}}
$$

und endlich aus (4.14)

$$
\begin{aligned}
L & \geqslant\left(2^{\frac{1}{2} \cdot \frac{\log T}{\log \log T}}\right)\left(\frac{\sqrt{T}}{\left|\frac{1}{2}+i 14,13 \ldots\right|^{\frac{\log T}{\log \log T}}}\right)|z| \\
& \geqslant \sqrt{T} e^{-5 \frac{\log T}{(\log \log T)^{2}}}, \quad T>c_{24} .
\end{aligned}
$$

Aus (4.13) und (4.21) ergibt sich

$$
\text { (4.22) } \quad \frac{\omega_{0}^{k}}{k !} \max _{\omega-\omega_{0} \leqslant \omega_{1} \leqslant \omega}\left|e^{\omega_{1} / 2} H\left(e^{\omega_{1} / 2}\right)\right| \geqslant \frac{1}{2} \sqrt{T} e^{-5 \frac{\log T}{(\log \log T)^{2}}} .
$$

Aus (3.3) und (3.4) folgt aber

$$
\frac{\omega_{0}^{k}}{k !} \leqslant \frac{\left(e \omega_{0}\right)^{k}}{k^{k}} \leqslant e^{2 \frac{\log T \log \log \log \log T}{\log \log T}} .
$$


Wir erhalten nun endlich

$$
\max _{\omega-\omega_{0} \leqslant \omega_{1} \leqslant \omega}\left|e^{\omega_{1} / 2} H\left(e^{\omega_{1} / 2}\right)\right| \geqslant \sqrt{T} e^{-8 \frac{\log T \log \log \log \log T}{\log \log T}}
$$

und daraus wegen (4.2), (3.2), (3.3) und der Definition von $H(\beta)$

für

$$
\max _{T e^{-\varphi(T)} \leqslant \beta \leqslant T}\left|\beta \sum_{1}^{\infty} \frac{\mu(n)}{n} e^{-(\beta / 2)^{2}}\right| \geqslant \sqrt{T} e^{-3 \frac{\log T \log \log \log \log T}{\log \log T}}
$$

$$
T>\max \left(c_{25}, e^{\sqrt{\alpha}}\right), \quad \varphi(T)=\frac{\log T \log \log \log T}{\log \log T} .
$$

Daraus bekommt man einfach (1.6) und damit ist unser Satz bewiesen.

\section{Literaturverzeichnis}

[1] G. H. Hardy and J. E. Littlewood, Contributions to the theory of the Riemann zeta-function and the theory of the distribution of primes, Acta Math. 41 (1918), pp. 117-196.

[2] S. Knapowski, On the Möbius function, Acta Arith. 4 (1958), pp. 209-216.

[3] E. Landau, Vorlesungen über Zahlentheorie, Bd. II, Leipzig 1927.

[4] N. Nielsen, Handbuch der Theorie der Gammafunktion, Leipzig 1906.

[5] E. C. Titchmarsh, The theory of the Riemann zeta-function, Oxford $195 \mathrm{I}$.

[6] P. Turán, Einę neue Methode in der Analysis und deren Anwendungen, Budapest 1953.

UNIWERS YTET IM. ADAMA MICKIEWICZA W POZNANIU

ADAM MTCKIEWICZ UNIVERSITÄT IN POZNAŃ

Rę̧u par la Rédaction le 12. 3. 1962

\section{Arithmetical notes, IX. \\ On the set of integers representable \\ as a product of a prime and a square}

by

E. CoHen (Knoxville, Tennessee)

1. Introduction. In this note we obtain an asymptotic determination of the number $A(x)$ of positive integers $n$ not exceeding $x$ which are expressible as the product of a prime by a square. In particular, we show that

$$
A(x) \sim \frac{\pi^{2}}{6} \cdot \frac{x}{\log x} \quad \text { as } \quad x \rightarrow \infty
$$

Actuailly, a more precise approximation to $A(x)$ is deduced (Theorem 2.1.) The proof is based upon the prime number theorem and a simple factorization principle (Remark, §2). Using the theorem of $\S 2$ on $A(x)$, a similar result is derived for a related problem in $\S 3$.

2. The main result. Let $A$ denote the set of all $n$ which can be represented as a prime multiplied by a square. Since every $n$ is uniquely expressible as a product of a square-free number and a square, we have immediately the

Remark. If $n$ is in $A$, then $n$ has a factorization $n=p Q, p$ prime, $Q$ square, and this representation is unique.

In addition to this remark we shall use the prime number theorem in the form

$$
\pi(x)=\frac{x}{\log x}+O\left(\frac{x}{\log ^{2} x}\right)
$$

where $\pi(x)$ denotes, as usual, the number of primes $\leqslant x$ (Landau [2], $\S 54)$.

THEOREM 2.1. If $x \geqslant 2$, then

$$
A(x)=\frac{\pi^{2}}{6} \cdot \frac{x}{\log x}+O\left(\frac{x}{\log ^{2} x}\right) .
$$

